\title{
Familial Barrett's Esophagus: Clues to Genetic Risks for Esophageal Adenocarcinoma
}

\author{
Joel H. Rubenstein
}

Published online: 3 April 2011

(C) Springer Science+Business Media, LLC (Outside the USA) 2011

Esophageal adenocarcinoma is a particularly deadly neoplasm, and the incidence of it has been rising at an alarming rate in recent decades in Western countries [1, 2]. Barrett's esophagus has been recognized as a precursor lesion for cancer, and screening and subsequent surveillance or other intervention for this lesion is an attractive proposition. Despite the rising incidence, the cancer still remains relatively uncommon. Therefore, selecting highrisk patients for screening, surveillance, or intervention is important in order to make the practice economically reasonable. Risk factors have been established for the cancer and for Barrett's esophagus, including male gender, white race, older age, symptoms of gastroesophageal reflux, obesity, tobacco use, and absence of infection with Helicobacter pylori. However, most of these factors are highly prevalent in Western societies, so more specific risk factors would improve the efficiency of clinical practice. The association with white race might be because of environmental differences among races, but also suggests that genetic factors play an important role.

Obviously, humans are not evolving rapidly enough to explain the rising incidence of esophageal adenocarcinoma, which is certainly because of relatively recent changes in environment or behavior. However, those changes in environment and behavior may interact with genetic factors, such that only genetically predisposed individuals

\footnotetext{
J. H. Rubenstein $(\bowtie)$

Veterans Affairs Center for Clinical Management Research, VA Medical Centre, 111-D, 2215 Fuller Road, Ann Arbor, MI 48105, USA

e-mail: jhr@med.umich.edu

J. H. Rubenstein

Division of Gastroenterology, University of Michigan Medical School, Ann Arbor, MI, USA
}

develop the cancer in the modern setting of obesity and low prevalence of Helicobacter pylori. Indeed, a family history of esophageal adenocarcinoma or Barrett's esophagus has been associated with the presence of both of those diagnoses [3]. A number of case reports of individual families suggest an autosomal dominant pattern of inheritance with incomplete penetrance [4-6].

Familial effects on the risk of esophageal adenocarcinoma may act via the promotion of other known risk factors (such as obesity or gastroesophageal reflux), via the promotion of Barrett's esophagus among those with the recognized risk factors, via the promotion of neoplastic transformation within Barrett's esophagus, or combinations of these loci of effects. In this issue of Digestive Diseases and Sciences, Ash and colleagues have begun to address these effects by studying their cohort of patients with Barrett's esophagus [7]. Records of nearly 1,000 patients with Barrett's esophagus were abstracted regarding their family history of either Barrett's esophagus or esophageal cancer, and those with a positive history were interviewed for details. Nearly $6 \%$ of patients with Barrett's esophagus were found to have a positive family history. The investigators then compared these familial cases of Barrett's esophagus with the non-familial cases, seeking differences in demographics or clinical characteristics. Of note, they found no difference in gender, race, smoking history, presence of Helicobacter pylori, hiatal hernia, or length of Barrett's esophagus. The investigators did find that familial cases were younger, and less likely to harbor neoplasia; however, they attribute these differences to referral bias, because patients with neoplasia are frequently referred to their center from outside practices, and patients with familial Barrett's esophagus without neoplasia may be more likely to be referred to them than other non-neoplastic Barrett's esophagus patients. The younger age of the familial Barrett's 
esophagus patients could be because of either the family history prompting them to undergo endoscopy at a younger age or the family history causing the development of Barrett's esophagus at a younger age. These findings suggest that the familial factors may not strongly affect the neoplastic progression of Barrett's esophagus, but rather promote the development of Barrett's esophagus.

The study by Ash and colleagues examines the effect of familiarity in Barrett's esophagus, but this does not necessarily mean that the effects are a consequence of genetic differences [7]. Familiarity can also be due to shared environment, for example tobacco habits, or eating and physical activity habits leading to obesity. Familiarity could also be due to inherited non-genetic factors, for example Helicobacter pylori or other infections. The findings in their study could potentially under-represent the effects of familiarity, particularly if subjects' family history status is misclassified, either because of small families and young relatives (reducing the likelihood of a family presenting with a diagnosis if the genes have low penetrance) or underdiagnosis of Barrett's esophagus because of mild symptoms, practice patterns of the relatives' physicians, or access to medical care. Nonetheless, in their large cohort of Barrett's esophagus patients, they found no solid evidence that clinical differences exist between familial and nonfamilial cases. This suggests that familial factors may lead to esophageal adenocarcinoma through promotion of the typical known risk factors for Barrett's esophagus.

The genetic factors that account for familial Barrett's esophagus are likely also to account for many cases of esophageal adenocarcinoma not recognized to arise from familial clusters. A number of studies have begun to examine whether single nucleotide polymorphisms (SNPs) of pertinent genes or their promoter regions are associated with Barrett's esophagus or esophageal adenocarcinoma. For instance, the TT genotype of the C609T SNP of the $\mathrm{NAD}(\mathrm{P}) \mathrm{H}$ :quinone oxidoreductase 1 gene was found to be associated with reduced risk of esophageal adenocarcinoma (odds ratio $=0.16,95 \%$ confidence intervals $=0.03,0.94$ ), but only $5 \%$ of controls with gastroesophageal reflux harbored that protective genotype $(95 \%$ of reflux sufferers would be classified as at increased risk for cancer) [8]. Polymorphisms that specifically identify a relatively small group of patients at risk of esophageal adenocarcinoma would be more useful to improve the efficiency of clinical practice. Such a SNP is the GG genotype of the rs6898743 SNP of the growth hormone receptor gene, which has been associated with fivefold increased odds of esophageal adenocarcinoma compared with population controls and found in only $3 \%$ of population controls [9]. However, only $10 \%$ of cancer cases harbored that genotype. Unfortunately, the greater specificity of that genotype comes at the expense of sensitivity for cancer.
As mentioned earlier, any effect of genetic factors on the risk of esophageal adenocarcinoma are likely to be affected by interaction with environmental or behavioral factors. Indeed, Zhai and colleagues found no overall effect of a SNP in the interleukin-1B gene (odds ratio for esophageal adenocarcinoma vs. controls $=1.2 ; 95 \%$ confidence inter$\mathrm{val}=0.51,2.6$ ), but found a very strong effect among subjects with symptoms of gastroesophageal reflux (odds ratio $=15.6 ; 95 \%$ confidence interval $=1.93,27.0$ ) $[10]$. However, fewer than $3 \%$ of cancer subjects would be identified by this SNP. Similar interactions with symptoms of gastroesophageal reflux have been described for SNPs of the DNA repair enzyme $\mathrm{O}^{6}$-methylguanine-DNA methyltransferase [11].

Sequencing the human genome has held the promise of personalized genomic medicine: the attractive notion that by sequencing each of our patients' genomes, we would have a crystal ball predicting which diseases each will develop and exactly how to intervene to prevent that fate. However, we are slowly finding that most of the classic type of diseases associated with mutations in a single gene have probably already been discovered [12]. As with most of the remaining diseases, the genetic effect on the development of esophageal adenocarcinoma is probably more complicated than a single dominant gene; more likely is that it involves the effects of multiple genes interacting with environmental conditions and with each other. Paradoxically, if the risk of cancer with any one of these genes is very high, then it is likely to account for only a small minority of cases of cancers, because of the rarity of the genotype. On the other hand, if a gene is associated with most cases of cancer, an individual with that genotype is likely to have only a very modest increased risk of cancer over those without the genotype. As a result, knowledge of these genetic associations are unlikely to be useful in the context of population screening or surveillance for cancer. They will be more useful for elucidating the biological pathways involved in the development of Barrett's esophagus and esophageal adenocarcinoma. Perhaps they may also be useful for predicting response to specific intervention, for example chemoprevention or endoscopic ablation. Many more studies are needed before we will know whether genetic associations with Barrett's esophagus and esophageal adenocarcinoma can be translated into meaningful personalized genomic medicine, and studying familial Barrett's esophagus can provide important clues to the etiology of Barrett's esophagus and esophageal adenocarcinoma.

\section{References}

1. Pohl H, Welch HG. The role of overdiagnosis and reclassification in the marked increase of esophageal adenocarcinoma incidence. J Natl Cancer Inst. 2005;97:142-146. 
2. Bollschweiler E, Wolfgarten E, Gutschow C, Holscher AH. Demographic variations in the rising incidence of esophageal adenocarcinoma in white males. Cancer. 2001;92:549-555.

3. Chak A, Lee T, Kinnard MF, et al. Familial aggregation of Barrett's oesophagus, oesophageal adenocarcinoma, and oesophagogastric junctional adenocarcinoma in Caucasian adults. Gut. 2002;51:323-328.

4. Munitiz V, Parrilla P, Ortiz A, Martinez-de-Haro LF, Yelamos J, Molina J. High risk of malignancy in familial Barrett's esophagus: presentation of one family. J Clin Gastroenterol. 2008;42: 806-809.

5. Jochem VJ, Fuerst PA, Fromkes JJ. Familial Barrett's esophagus associated with adenocarcinoma. Gastroenterology. 1992;102: 1400-1402.

6. Crabb DW, Berk MA, Hall TR, Conneally PM, Biegel AA, Lehman GA. Familial gastroesophageal reflux and development of Barrett's esophagus. Ann Intern Med. 1985;103:52-54.

7. Ash S, Vaccaro BJ, Dabney MK, Chung WK, Lightdale CJ, Abrams JA. Comparison of endoscopic and clinical characteristics of patients with familial and sporadic Barrett's esophagus. Dig Dis Sci. 2011 (Epub ahead of print). doi:10.1007/s10620011-1620-3.
8. di Martino E, Hardie LJ, Wild CP, et al. The NAD(P)H:quinone oxidoreductase I C609T polymorphism modifies the risk of Barrett esophagus and esophageal adenocarcinoma. Genet Med. 2007;9:341-347.

9. McElholm AR, McKnight A-J, Patterson CC, Johnston BT, Hardie LJ, Murray LJ, et al. A population-based study of IGF axis polymorphisms and the esophageal inflammation, metaplasia, adenocarcinoma sequence. Gastroenterology. 2010;139:204212.e3.

10. Zhai R, Chen F, Liu G, et al. Interactions among genetic variants in apoptosis pathway genes, reflux symptoms, body mass index, and smoking indicate two distinct etiologic patterns of esophageal adenocarcinoma. J Clin Oncol. 2010;28:2445-2451.

11. Doecke J, Zhao ZZ, Pandeya N, et al. Polymorphisms in MGMT and DNA repair genes and the risk of esophageal adenocarcinoma. Int J Cancer. 2008;123:174-180.

12. Feero WG, Guttmacher AE, Manolio TA. Genomewide association studies and assessment of the risk of disease. $N$ Engl J Med. 2010;363:166-176. 